\title{
ON THE DEGREE OF APPROXIMATION BY MODIFIED GAMMA OPERATORS
}

\section{GRAŻYNA KRECH}

Received 06 May, 2014

\begin{abstract}
We consider certain linear operators associated with gamma function in polynomial weighted spaces of functions of one variable and study approximation properties of these operators, including theorems on the degree of approximation.
\end{abstract}

2010 Mathematics Subject Classification: 41A25; 41A36

Keywords: gamma type operator, linear operators, approximation order

\section{INTRODUCTION}

In [11] Lupas and Müller introduced the sequence of linear positive operators $\left\{G_{n}\right\}$ defined by

$$
G_{n}(f ; x)=\int_{0}^{\infty} g_{n}(x, u) f\left(\frac{n}{u}\right) d u
$$

which is called gamma operator, where $g_{n}(x, u)=\frac{x^{n+1}}{n !} e^{-x u} u^{n}, x \in \mathbb{R}_{+}:=(0, \infty)$. Approximation problems for $G_{n}$ in some function spaces were examined in many papers, for example in $[1,11,13,15]$.

The above operators were modified by several authors (e.g. [3, 4, 12]) which showed that new operators have similar approximation properties to $G_{n}$ (see $[2,5$, $8-10,14])$.

The approximation of functions by gamma type operators

$$
L_{n}(f ; x)=\frac{(2 n+3) ! x^{n+3}}{n !(n+2) !} \int_{0}^{\infty} \frac{t^{n}}{(x+t)^{2 n+4}} f(t) d t, \quad x \in \mathbb{R}_{+}
$$

in polynomial weighted spaces $C_{p}$ were studied by Karsli [6]. The space $C_{p}, p \in$ $\mathbb{N}_{0}:=\mathbb{N} \cup\{0\}$, is associated with the weight function

$$
w_{0}(x)=1 \quad \text { and } \quad w_{p}(x)=\frac{1}{1+x^{p}}, \quad p \in \mathbb{N},
$$


and consists of all real-valued functions $f$ for which $f w_{p}$ is uniformly continuous and bounded on $\mathbb{R}_{0}=[0, \infty)$. The norm on $C_{p}$ is defined by

$$
\|f\|_{p}=\sup _{x \in \mathbb{R}_{0}} w_{p}(x)|f(x)| .
$$

Moreover, if $f$ is right-side continuous at $x=0$, we define $L_{n}(f ; 0)=f(0), n \in \mathbb{N}$.

We shall use the modulus of continuity of $f \in C_{p}$,

$$
\omega_{p}(f, \delta)=\sup _{h \in[0, \delta]}\left\|\Delta_{h} f\right\|_{p}, \quad \delta \geq 0,
$$

and the modulus of smoothness of $f \in C_{p}$,

$$
\omega_{p}^{2}(f, \delta)=\sup _{h \in[0, \delta]}\left\|\Delta_{h}^{2} f\right\|_{p}, \quad \delta \geq 0,
$$

where

$$
\Delta_{h} f(x)=f(x+h)-f(x), \quad \Delta_{h}^{2} f(x)=f(x+2 h)-2 f(x+h)+f(x)
$$

for $x, h \in \mathbb{R}_{0}$.

In [6], it was showed that $L_{n}$ defines a positive linear operator $C_{p} \rightarrow C_{p}$. For $f \in C_{p}, p \in \mathbb{N}_{0}$ and $x \in(0, \infty)$, it was proved that

$$
w_{p}(x)\left|L_{n}(f ; x)-f(x)\right| \leq M_{p} \omega_{p}^{2}\left(f, \frac{x}{\sqrt{n+2}}\right)+\omega_{p}\left(f, \frac{x}{n+2}\right),
$$

where $M_{p}$ is a positive constant.

From (1.2) we conclude that if $f \in C_{p}, p \in \mathbb{N}_{0}$, then

$$
\left\|L_{n}(f ; \cdot)-f\right\|_{p}=O\left(n^{-1 / 2}\right) .
$$

Thus the question arises, whether the rate of approximation given in the paper [6] cannot be improved. In connection with this question we propose a new family of linear operators. The method was inspired by Kirov [7].

Let $D_{p}, p \in \mathbb{N}$, be the set of all real-valued continuous functions $f$ on $\mathbb{R}_{+}$, such that

$$
w_{p}(x) x^{k} f^{(k)}(x), \quad w_{p-k}(x) f^{(k)}(x), \quad k=0,1,2, \ldots, p
$$

are continuous and bounded on $\mathbb{R}_{+}$, and $f^{(p)}$ is uniformly continuous on $\mathbb{R}_{+}$. The norm on $D_{p}$ is given by (1.1).

We introduce the following class of operators in $D_{p}, p \in \mathbb{N}$.

Definition 1. Fix $p \in \mathbb{N}$. For functions $f \in D_{p}$ we define the operators

$$
A_{n}(f ; p ; x)=\int_{0}^{\infty} \frac{(2 n+3) ! x^{n+3} t^{n}}{n !(n+2) !(x+t)^{2 n+4}} \sum_{j=0}^{p} \frac{f^{(j)}(t)(x-t)^{j}}{j !} d t,
$$

$x \in \mathbb{R}_{+}, n \in \mathbb{N}$. 
Throughout this paper we shall denote by $M_{\alpha, \beta}$ positive constants depending only on indicated parameters $\alpha, \beta$, and point out that they are not the same at each occurrence.

\section{Auxiliary RESUlts}

In this section we give some preliminary results which will be used in the rest part of this paper.

In the sequel the following functions will be meaningful:

$$
e_{m}(t)=t^{m}, \quad \phi_{x, m}(t)=(t-x)^{m}, \quad m \in \mathbb{N}_{0}, x, t \in \mathbb{R}_{0} .
$$

Using the definition (1.3) and the equality

$$
\left(x^{n}\right)^{(k)}=\frac{n !}{(n-k) !} x^{n-k},
$$

we can prove the following lemma.

Lemma 1. Fix $p \in \mathbb{N}_{0}$. Then we have

$$
\begin{gathered}
A_{n}\left(e_{0} ; p ; x\right)=1, \\
A_{n}\left(e_{m} ; p ; x\right)=e_{m}(x) \quad \text { for } \quad p \leq m .
\end{gathered}
$$

In [4] the author obtained the following result.

Lemma 2 ([4]). For any $m \in \mathbb{N}_{0}, m \leq n+2$, we have

$$
L_{n}\left(e_{m} ; x\right)=\frac{(n+m) !(n+2-m) !}{n !(n+2) !} x^{m}
$$

and there exists a positive constant $M_{m}, m \in \mathbb{N}_{0}$, such that

$$
L_{n}\left(\phi_{x, m} ; x\right) \leq M_{m} \frac{x^{m}}{n^{[(m+1) / 2]}},
$$

where $[a]$ denotes the integral part of $a$.

Lemma 3. Fix $p \in \mathbb{N}_{0}$. Then there exists a positive constant $M_{p}$ such that

$$
\left\|L_{n}\left(1 / w_{p} ; x\right)\right\|_{p} \leq M_{p}, \quad n \in \mathbb{N} .
$$

Proof. For $p=0$ the inequality (2.4) is obvious.

Let $p \in \mathbb{N}$. Using (2.2) we obtain

$$
\omega_{p}(x)\left|L_{n}\left(1 / \omega_{p} ; x\right)\right|=\frac{1}{1+x^{p}}+\frac{1}{1+x^{p}} \cdot \frac{(n+p) !(n+2-p) !}{n !(n+2) !} x^{p} \leq M_{p},
$$

which gives the assertion.

Similarly we can prove 
Lemma 4. Fix $p \in \mathbb{N}_{0}$. Then there exists a positive constant $M_{p}$ such that

$$
\sup _{x \in \mathbb{R}_{+}} w_{p}(x) x^{k} L_{n}\left(1 / w_{p-k} ; x\right) \leq M_{p}, \quad n \in \mathbb{N}, k=0,1,2, \ldots, p .
$$

Next we shall prove the following result.

Lemma 5. Fix $p \in \mathbb{N}$. Then there exists a positive constant $M_{f, p}$ such that

$$
\left\|A_{n}(f ; p ; \cdot)\right\|_{p} \leq M_{f, p}
$$

for all $f \in D_{p}$ and $n \in \mathbb{N}$.

Formulas (1.3) and (2.6) show that $A_{n}(f ; p)$ is well-defined on the space $D_{p}$, $p \in \mathbb{N}$.

Proof. Let $f \in D_{p}, p \in \mathbb{N}$. From this, using the elementary inequality

$$
(a+b)^{k} \leq 2^{k-1}\left(a^{k}+b^{k}\right), \quad a, b \geq 0, k \in \mathbb{N}_{0},
$$

we obtain

$$
|x-t|^{k}\left|f^{(k)}(t)\right| \leq 2^{k-1}\left|f^{(k)}(t)\right|\left(x^{k}+t^{k}\right) \leq M_{f, p, k}\left(\frac{1}{w_{p}(t)}+\frac{x^{k}}{w_{p-k}(t)}\right),
$$

$x, t \in \mathbb{R}_{+}, k=0,1,2, \ldots, p$. By linearity of $A_{n}$ we have

$$
\begin{aligned}
& w_{p}(x)\left|A_{n}(f ; p ; x)\right| \\
& \quad \leq M_{f, p} w_{p}(x) \int_{0}^{\infty} \frac{(2 n+3) ! x^{n+3} t^{n}}{n !(n+2) !(x+t)^{2 n+4}}\left\{\frac{1}{w_{p}(t)}+\sum_{j=0}^{p} \frac{x^{j}}{w_{p-j}(t)}\right\} d t \\
& \quad=M_{f, p} w_{p}(x)\left\{L_{n}\left(1 / w_{p} ; x\right)+\sum_{j=0}^{p} x^{j} L_{n}\left(1 / w_{p-j} ; x\right)\right\} .
\end{aligned}
$$

Thus, using (2.4) and (2.5) we conclude that

$$
w_{p}(x)\left|A_{n}(f ; p ; x)\right| \leq M_{f, p},
$$

which gives the result.

\section{MAin RESUlts}

In this section we give theorems on the degree of approximation of the function $f \in D_{p}, p \in \mathbb{N}$, by the operators $A_{n}(f ; p)$.

Theorem 1. Fix $p \in \mathbb{N}_{0}$. Then there exists a positive constant $M_{p}$ such that, for every $f \in D_{2 p+1}$, we have

$$
\left\|A_{n}(f ; 2 p+1 ; \cdot)-f\right\|_{2 p+1} \leq \frac{M_{p}}{n^{p+\frac{1}{2}}}\left\|f^{(2 p+1)}\right\|_{0}, \quad n \in \mathbb{N} .
$$


Proof. Let $f \in D_{2 p+1}, p \in \mathbb{N}_{0}$. This implies that $f^{(2 p+1)} \in C_{0}$.

Observe that we can write

$$
\begin{aligned}
f(x)= & \sum_{j=0}^{2 p+1} \frac{f^{(j)}(t)(x-t)^{j}}{j !} \\
& +\frac{(x-t)^{2 p+1}}{(2 p) !} \int_{0}^{1}(1-s)^{2 p}\left\{f^{(2 p+1)}(t+s(x-t))-f^{(2 p+1)}(t)\right\} d s .
\end{aligned}
$$

Using (2.1) and (1.3), we obtain

$$
\begin{aligned}
& w_{2 p+1}(x)\left|A_{n}(f ; 2 p+1 ; x)-f(x)\right| \\
& \leq w_{2 p+1}(x) \int_{0}^{\infty} \frac{(2 n+3) ! x^{n+3} t^{n}}{n !(n+2) !(x+t)^{2 n+4}}\left|\sum_{j=0}^{2 p+1} \frac{f^{(j)}(t)(x-t)^{j}}{j !}-f(x)\right| d t \\
& \leq w_{2 p+1}(x) \int_{0}^{\infty} \frac{(2 n+3) ! x^{n+3} t^{n}}{n !(n+2) !(x+t)^{2 n+4}} \cdot \frac{|x-t|^{2 p+1}}{(2 p) !} \\
& \quad \times \int_{0}^{1}(1-s)^{2 p}\left\{\left|f^{(2 p+1)}(t+s(x-t))\right|+\left|f^{(2 p+1)}(t)\right|\right\} d s d t .
\end{aligned}
$$

By our assumption we have

$$
\begin{aligned}
& w_{2 p+1}(x)\left|A_{n}(f ; 2 p+1 ; x)-f(x)\right| \\
& \quad \leq M_{p} w_{2 p+1}(x)\left\|f^{(2 p+1)}\right\|_{0} \int_{0}^{\infty} \frac{(2 n+3) ! x^{n+3} t^{n}}{n !(n+2) !(x+t)^{2 n+4}} \cdot \frac{|x-t|^{2 p+1}}{(2 p+1) !} d t \\
& \quad \leq M_{p} w_{2 p+1}(x)\left\|f^{(2 p+1)}\right\|_{0} L_{n}\left(\left|\phi_{x, 2 p+1}\right| ; x\right) .
\end{aligned}
$$

Moreover, by the Cauchy-Schwarz inequality and using (2.3), we get

$$
\begin{aligned}
w_{2 p+1}(x) L_{n}\left(\left|\phi_{x, 2 p+1}\right| ; x\right) & \leq w_{2 p+1}(x)\left(L_{n}\left(\phi_{x, 4 p+2} ; x\right)\right)^{1 / 2} \\
& \leq\left(M_{p} \frac{x^{4 p+2}}{\left(1+x^{2 p+1}\right)^{2}} \cdot \frac{1}{n^{[(4 p+3) / 2]}}\right)^{1 / 2} \\
& \leq \frac{M_{p}}{n^{p+\frac{1}{2}}} .
\end{aligned}
$$

Combining (3.2) with (3.1) we immediately conclude

$$
\left\|A_{n}(f ; 2 p+1 ; \cdot)-f\right\|_{2 p+1} \leq \frac{M_{p}}{n^{p+\frac{1}{2}}}\left\|f^{(2 p+1)}\right\|_{0}
$$

for $n \in \mathbb{N}$ and $p \in \mathbb{N}_{0}$. The theorem is proved. 
Theorem 2. Fix $p \in \mathbb{N}_{0}$. Then there exists a positive constant $M_{p}$ such that, for every $f \in D_{2 p+2}$, we have

$$
\left\|A_{n}(f ; 2 p+2 ; \cdot)-f\right\|_{2 p+2} \leq \frac{M_{p}}{n^{p+1}}\left\|f^{(2 p+2)}\right\|_{0}, \quad n \in \mathbb{N} .
$$

Proof. Let $f \in D_{2 p+2}, p \in \mathbb{N}_{0}$. Then $f^{(2 p+2)} \in C_{0}$. Similarly as in the proof of Theorem 1, we can write

$$
\begin{aligned}
& w_{2 p+2}(x)\left|A_{n}(f ; 2 p+2 ; x)-f(x)\right| \\
& \leq w_{2 p+2}(x) \int_{0}^{\infty} \frac{(2 n+3) ! x^{n+3} t^{n}}{n !(n+2) !(x+t)^{2 n+4}} \cdot \frac{(x-t)^{2 p+2}}{(2 p+1) !} \\
& \quad \times \int_{0}^{1}(1-s)^{2 p+1}\left\{\left|f^{(2 p+2)}(t+s(x-t))\right|+\left|f^{(2 p+2)}(t)\right|\right\} d s d t .
\end{aligned}
$$

From this, by our assumption and using (2.3), we obtain

$$
\begin{aligned}
w_{2 p+2}(x)\left|A_{n}(f ; 2 p+2 ; x)-f(x)\right| \\
\quad \leq M_{p} w_{2 p+2}(x)\left\|f^{(2 p+2)}\right\|_{0} \int_{0}^{\infty} \frac{(2 n+3) ! x^{n+3} t^{n}}{n !(n+2) !(x+t)^{2 n+4}}(x-t)^{2 p+2} d t \\
\quad=M_{p} w_{2 p+2}(x)\left\|f^{(2 p+2)}\right\|_{0} L_{n}\left(\phi_{x, 2 p+2} ; x\right) \\
\quad \leq M_{p}\left\|f^{(2 p+2)}\right\|_{0} \frac{x^{2 p+2}}{1+x^{2 p+2}} \cdot \frac{1}{n^{[(2 p+3) / 2]}} \leq \frac{M_{p}}{n^{p+1}}\left\|f^{(2 p+2)}\right\|_{0}
\end{aligned}
$$

for $x \in \mathbb{R}_{+}, n \in \mathbb{N}$ and $p \in \mathbb{N}_{0}$. This completes the proof of Theorem 2 .

Corollary 1. For every fixed $f \in D_{p}, p \in \mathbb{N}$ we have

$$
\left\|A_{n}(f ; p ; \cdot)-f\right\|_{p}=O\left(n^{-p / 2}\right) .
$$

Remark 1 . Corollary 1 shows that the operators $A_{n}, n \in \mathbb{N}$, give a better order of approximation of functions $f \in D_{p}, p \in \mathbb{N}$, than $L_{n}$.

\section{REFERENCES}

[1] W. Chen and S. Guo, "On the rate of convergence of the Gamma Operator for functions of bounded variation," Approx. Theory Appl., vol. 1, no. 5, pp. 85-96, 1985.

[2] A. İzgi, "Voronovskaya type asymptotic approximation by modified Gamma operators," Appl. Math. Comput., vol. 217, pp. 8061-8067, 2011, doi: 10.1016/j.amc.2011.03.005.

[3] A. İzgi and I. Büyükyazici, "Approximation and rate of approximation on unbounded intervals," Kastamonu Edu. Journal Okt., vol. 11, no. 2, pp. 451-460, 2003.

[4] H. Karsli, "Rate of convergence of a new Gamma Type Operators for functions with derivatives of bounded variation," Math. Comput. Modelling, vol. 45, no. 5-6, pp. 617-624, 2007, doi: 10.1016/j.mcm.2006.08.001.

[5] H. Karsli, V. Gupta, and A. İzgi, "Rate of pointwise convergence of a new kind of gamma operators for functions of bounded variation," Appl. Math. Letters, vol. 22, no. 4, pp. 505-510, 2009, doi: 10.1016/j.aml.2006.12.015. 
[6] H. Karsli and M. A. Özarslan, "Direct local and global approximation results for operators of Gamma type," Hacettepe Journal of Mathematics and Statistics, vol. 39, no. 2, pp. 241-253, 2010.

[7] G. H. Kirov, "A generalization of the Bernstein polynomials," Mathematica Balkanica, vol. 6, no. 2, pp. 147-153, 1992.

[8] G. Krech, "A note on the paper "Voronovskaya type asymptotic approximation by modified Gamma operators"," Appl. Math. Comput., vol. 219, pp. 5787-5791, 2013, doi: 10.1016/j.amc.2012.11.100.

[9] G. Krech, "Modified gamma operators in Lp spaces," Lith. Math. J., vol. 54, pp. 454-462, 2014, doi: 10.1007/s10986-014-9256-6.

[10] G. Krech, "On the rate of convergence for modified Gamma operators," Rev. Un. Mat. Argentina, vol. 55, pp. 123-131, 2014.

[11] A. Lupas and M. M. Müller, "Approximationseigenschafen der Gammaoperatoren," Math. Zeischr., vol. 98, pp. 208-226, 1967.

[12] S. M. Mazhar, "Approximation by positive operators on infinite intervals," Mathematica Balkanica, vol. 5, no. 2, pp. 99-104, 1991.

[13] V. Totik, "The Gamma operators in $L_{p}$ spaces," Publ. Math., vol. 32, pp. 43-55, 1985.

[14] X.-W. Xu and J. Wang, "Approximation properties of modified Gamma operators," J. Math. Anal. Appl., vol. 332, pp. 798-813, 2007, doi: 10.1016/j.jmaa.2006.10.065.

[15] X.-M. Zeng, "Approximation properties of Gamma operators," J. Math. Anal. Appl., vol. 311, no. 2, pp. 389-401, 2005, doi: 10.1016/j.jmaa.2005.02.051.

\section{Author's address}

\section{Grażyna Krech}

AGH University of Science and Technology, Faculty of Applied Mathematics, Mickiewicza 30, 30-059 Kraków, Poland

E-mail address: grazynakrechegmail.com 